\title{
Accidente isquémico transitorio secundario a excrecencia de Lambl. Descripción de un caso y revisión de la literatura
}

\author{
J. R. SILES RUBIO, J. RUIZ DE CASTROVIEJO DEL CAMPO', R. TIRADO \\ MIRANDA $^{2}$, S. JANSEN CHAPARRO ${ }^{2}$, D. PAVLOVIC ${ }^{1}$ \\ Unidad de Cardiología. Hospital Costa del Sol. Marbella, Málaga. ${ }^{1}$ Unidad de \\ Cardiología. ${ }^{2}$ Servicio de Medicina Interna. Hospital Infanta Margarita. Cabra, Córdoba
}

TRANSIENT ISCHEMIC ATTACK DUE TO LAMBL'S EXCRECENCE. REPORT OF A CASE AND REVIEW OF THE LITERATURE

\section{RESUMEN}

Describimos el caso de un varón de 42 años, sin factores de riesgo cardiovasculares conocidos que presentó un accidente isquémico transitorio. Se descartó enfermedad sistémica y sustrato de hipercoagulabilidad como causa del mismo. Sólo mediante un estudio ecocardiográfico transesofágico se pudo confirmar una excrecencia de Lambl localizada en la válvula aórtica (strand valvular). Se realiza una revisión acerca del manejo clínico y las nuevas opciones terapéuticas en la prevención del ictus de esta inusual fuente cardioembólica.

PALABRAS CLAVE: Ictus. Accidente isquémico transitorio. Strand valvular. Embolismo cardiaco.

\section{ABSTRACT}

We describe a 42-year-old man presenting in the emergengy departament with transient ischemic attack without cardiovascular risk factors. Systemic disease and hypercoagulability substract was discarded. Only transesophageal echocardiography could confirm the presence of a Lambl's excrescence in the aortic valve (valvular strand).

We review clinical management and new therapeutic options in stroke prevention used in this unusual source of cardiac embolism.

KEY WORDS: Stroke. Transient ischemic attack. valvular strand. Cardiac embolism.

Siles Rubio JR, Ruiz de Castroviejo del Campo J, Tirado Miranda R, Jansen Chaparro S, Pavlovic D. Accidente isquémico transitorio secundario a excrecencia de Lambl. Descripción de un caso y revisión de la literatura. An Med Interna (Madrid) 2006; 23: 181-183.

\section{INTRODUCCIÓN}

En los pacientes con ictus isquémico, tras un estudio etiológico exhaustivo, existe un porcentaje significativo en los que no se evidencia la causa del mismo. La generalización de la ecocardiografía transesofágica como parte de la batería diagnóstica, ha permitido caracterizar nuevas fuentes cardioembólicas, que han venido a sumarse a las clásicas. Entre ellas se encuentran las excrecencias de Lambl o strands valvulares. Aunque su primera descripción data de 1856, en el momento actual no se han definido guías específicas para su tratamiento, realizándose habitualmente éste en base a análisis retrospectivos de estudios llevados a cabo en pacientes con eventos isquémicos cerebrales.

\section{CASO APORTADO}

Se trata de un varón de 42 años sin antecedentes médicos de interés. Deambula diariamente una hora, no es hipertenso, dislipémico, ni fumador y niega la ingesta de alcohol u otras drogas. No presenta antecedentes familiares directos relevantes.

Fue valorado en nuestro centro al presentar de forma brusca paresia del miembro superior derecho y disartria, las cuales se autolimitaron en veinte minutos. El paciente no refirió otra sintomatología. En la exploración física general no había datos relevantes, siendo su tensión arterial de 140/80 mmHg. La exploración neurológica, al margen del déficit motor del momento agudo, fue rigurosamente normal.

No se encontraron alteraciones patológicas en las pruebas de imagen craneales realizadas (radiografía de cráneo, tomografía

Trabajo aceptado: 7 de noviembre de 2005 
computarizada con contraste y angioresonancia magnética), en el electroencefalograma, ni en los potenciales evocados. La radiografía de tórax y el ecocardiograma transtorácico fueron normales. El Doppler arterial de troncos supraaórticos no evidenció placas ateroscleróticas. El estudio analítico (bioquímica general, lípidos, perfil hepático, función renal, estudio de coagulación, VSG, PCR, proteinograma, sedimento urinario, hemograma, serología luética, anticuerpos antinucleares, y factor reumatoide) no mostró valores patológicos. Asimismo, con el fin de descartar estados de hipercoagulabilidad, se realizó una determinación de anticuerpos antifosfolípidos, factor $\mathrm{V}$, antitrombina III, mutación G20210A de la protrombina, homocisteina, déficit de proteinas S y $\mathrm{C}$, así como la resistencia a la proteína $\mathrm{C}$ activada. En ninguno de ellos se hallaron valores patológicos.

Se realizó una ecocardiografía transesofágica (ETE) constatando la ausencia de permeabilidad del foramen oval de manera basal, mediante la utilización de contraste y tras la realización de la maniobra de Valsalva. No se evidenciaron placas ateroscleróticas en el trayecto visible de la aorta. Se visualizó una imagen filamentosa, fina y móvil de $9 \mathrm{~mm}$, visible tanto en la cara arterial como en la cara ventricular de la válvula aórtica. Ante estos hallazgos compatibles con un strand valvular aórtico se inició tratamiento antiagregante con $200 \mathrm{mg}$. diarios de ácido acetilsalicílico. En el seguimiento ambulatorio realizado durante el primer año, el paciente ha permanecido asintomático y la ETE realizada al final de este periodo confirmó la permanencia de la excrecencia valvular.

\section{DISCUSIÓN}

En pacientes con ictus isquémicos existe un porcentaje en los que no se puede establecer su etiología (1). En los últimos años hemos asistido a una disminución de los ictus criptogénicos, en parte debido a la mejora de las técnicas diagnósticas, así como a la aplicación de registros clínicos para los eventos cerebrovasculares (2). A este respecto, la generalización de la ETE (3) en el estudio de las fuentes cardioembólicas ha servido para identificar alteraciones que se suman a las clásicas causas de embolismos (4) (trombos y tumores intracavitarios, ecocontraste espontáneo, placas aórticas, valvulopatía mitral o persistencia de foramen oval permeable, entre otras) (3,5). Los strands valvulares (6) o

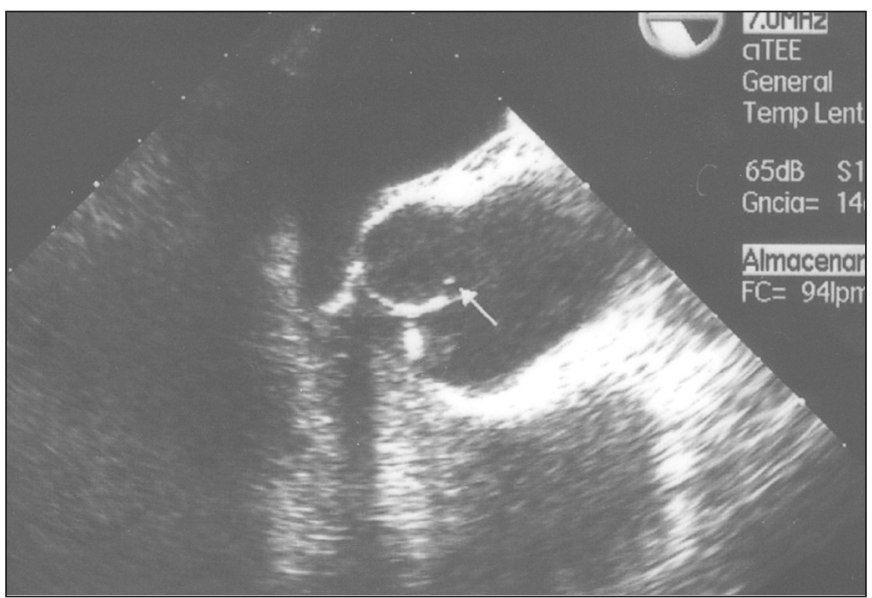

Fig. 1. Vista transesofágica multiplano a $120^{\circ}$ alineado con el eje largo del ventrículo izquierdo evidenciándose una imagen filamentosa de $9 \mathrm{~mm}$. en la cara arterial de la válvula aórtica. excrecencias de Lambl son estructuras que pueden adoptar formas filamentosas o laminares caracterizadas por su gran movilidad. Se encuentran recubiertas de fibrina y son resultado del estrés crónico al que están sometidas las válvulas cardiacas, tanto las nativas como las protésicas, siendo su localización más frecuente en la válvula mitral (6).

El diagnóstico diferencial de estas estructuras se establece, fundamentalmente, con las diferentes formas de endocarditis (infecciosas sobre válvulas nativas y protésicas, sobre marcapasos y las marasmáticas) y con los fibroelastomas papilares (6).

La importancia de la detección de estas estructuras radica en que su visualización en la ETE se asocia a embolizaciones sistémicas $(7,8)$.

Aunque en nuestro medio, la hipertensión arterial y la fibrilación auricular son los factores de riesgo más prevalentes en pacientes mayores con ictus (9), la asociación entre los strands y las embolizaciones con origen cardiaco es más fecuente en los pacientes menores de 50 años (10). La presencia de factores de riesgo como diabetes mellitus, tabaquismo y dislipemia son más comunes en pacientes sin strands, sugiriendo que dichos factores de riesgo no se asocian con los mismos (10).

Todo lo anterior hace que los strands valvulares deban ser considerados entre las posibilidades etiológicas de los cardioembolismos, fundamentalmente, en pacientes jóvenes.

La mayor aproximación etiológica a este grupo de fuentes cardioembólicas (11) ha planteando, a su vez, nuevos interrogantes respecto a su adecuado manejo terapéutico. Las tasas de recurrencia de eventos isquémicos en pacientes con strands valvulares, no ha sido claramente definida al no existir estudios randomizados que evaluen la eficacia de la terapéutica antitrombótica en estos pacientes(12). Un análisis del estudio PICSS (Patent foramen oval in cryptogenic stroke study) reveló, en relación al tratamiento, que no había diferencias significativas al comparar la efectividad de aspirina con warfarina en la prevención de eventos isquémicos recurrentes en pacientes con strands valvulares (12).

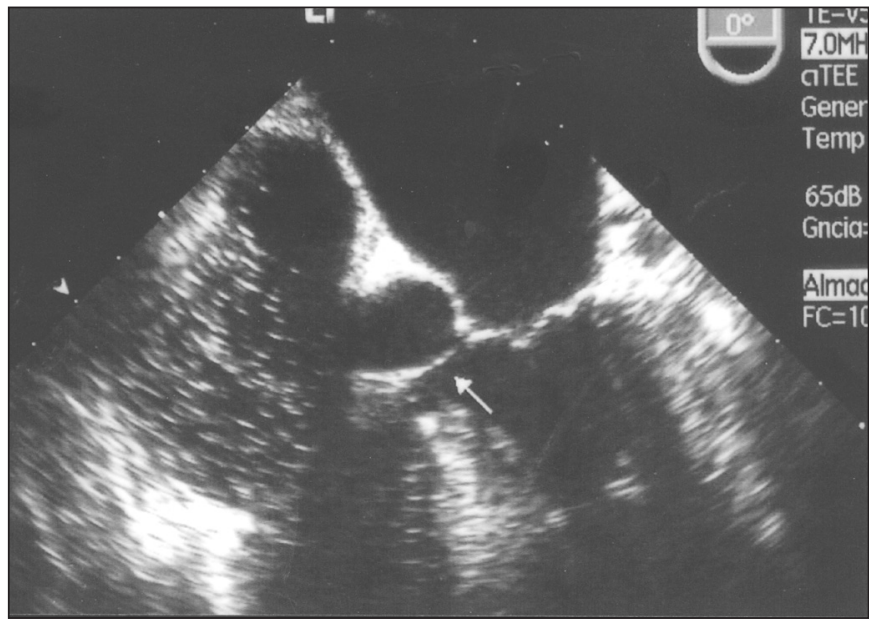

Fig. 2. Se aprecia la misma estructura de carácter filamentoso con gran movilidad en el tracto de salida ventricular izquierdo. 


\section{Bibliografía}

1. Sacco RL, Ellemberg J, Mohr JP, et al. Infarction of undetermined cause: the NICDS Stroke Data Bank. Ann Neurol 1989; 25: 382-390.

2. Arboix A, Oliveres M, Massons J, García-Eroles L, Bechich S, Targa C. Clinical and care implications of the 10-year application of a cerebrovascular diseases register. Descriptive analysis of the last 100 patients and comparative with the first 100 patients. Med Clin (Barc) 1998; 119: 286-289.

3. De Rook FA, Comess KA, Albers GW, Popp RL. Transesophageal echocardiography in the evaluation of stroke. Ann Intern Med 1992; 177: 922-932.

4. Vahedi K, Amarenco P Cardiac causes of stroke. Curr Treat Options Neurol 2000; 2: 305-18.

5. Palazzuoli A, Ricci D, Lenzi C, Lenzi J, Palazzzouli V. Transesophageal echocardiography for identifying potencial cardicac sources of embolism in patients with stroke. Neurol Sci 2000; 21: 195-202.

6. Voros S, Nanda NC, Thakur AC, Winokur TS, Samal AK. Lambl's excrescences (valvular strands). Echocardiography 1999; 16: 399-414.

7. Freedberg RS, Goodkin GM, Pérez JL, Tunick PA, Kronzon I. Valve strands are strongly associated with systemic embolization: a transesophageal echocardiographic study. J Am Coll Cardiol 1995; 26: 1709-12.

8. Nighoghossian N, Derex L, Perinetti M, Honnorat J, Barthelet M, Loire R Course of valvular strands in patients with stroke: cooperative study with transesophageal echocardiography. Am Heart J 1998; 136: 1065-9.

9. Acha O, Hernández JL, Penado S, Cano M, Riancho JA. Factores de riesgo e ictus en pacientes de diferentes edades. Rev Clin Esp 2003; 203: $189-92$.

10. Roberts JK, Omarali I, Di Tullio MR, Sciacca RR, Sacco RL, Homma S. Valvular strands and cerebral ischemia. Effect of demographics and strand characteristics. Stroke 1997; 28: 2185-8.

11. García Fernández MA, Bermejo J, Moreno M. Utilidad de las técnicas de imagen en la valoración etiológica y toma de decisiones en el paciente con ictus. Rev Esp Cardiol 2003; 56: 1119-32.

12. Homma S, Di tullio MR, Sciacca RR, Sacco RL, Mohr JP. Effect of aspirin and warfarin theraphy in stroke patients with valvular strands. Stroke 2004; 35: 1436-42 